See discussions, stats, and author profiles for this publication at: https://www.researchgate.net/publication/317801189

\title{
Automated EEG Signals Analysis Using Quantile Graphs
}

Conference Paper · May 2017

DOI: 10.1007/978-3-319-59147-6_9

CITATION

1

3 authors, including:

Andriana S. L. O. Campanharo

São Paulo State University

8 PUBlicATIONS 301 CITATIONS

SEE PROFILE

Some of the authors of this publication are also working on these related projects:

Multifractal analysis View project

REDD-PAC project View project
READS

111

Fernando Manuel Ramos

National Institute for Space Research, Brazil

206 PUBLICATIONS 1,676 CITATIONS

SEE PROFILE 


\title{
Automated EEG Signals Analysis Using Quantile Graphs
}

\author{
Andriana S.L.O. Campanharo ${ }^{1(凶)}$, Erwin Doescher ${ }^{2}$, and Fernando M. Ramos ${ }^{3}$ \\ 1 Departamento de Bioestatística, Instituto de Biociências, \\ Universidade Estadual Paulista, São Paulo, Botucatu, Brazil \\ andriana@ibb.unesp.br \\ 2 Departamento de Ciência e Tecnologia, Universidade Federal de São Paulo, \\ Campus São José dos Campos, São Paulo, Brazil \\ doescher@unifesp.br \\ 3 Laboratório de Computação e Matemática Aplicada, \\ Instituto Nacional de Pesquisas Espaciais, São José dos Campos, São Paulo, Brazil \\ fernando.ramos@inpe.br
}

\begin{abstract}
Recently, a map from time series to networks has been proposed $[7,8]$, allowing the use of network statistics to characterize time series. In this approach, time series quantiles are naturally mapped into nodes of a graph. Networks generated by this method, called Quantile Graphs (QGs), are able to capture and quantify features such as longrange correlations or randomness present in the underlying dynamics of the original signal. Here we apply the QG method to the problem of detecting the differences between electroencephalographic time series (EEG) of healthy and unhealthy subjects. Our main goal is to illustrate how the differences in dynamics are reflected in the topology of the corresponding QGs. Results show that the QG method cannot only differentiate epileptic from normal data, but also distinguish the different abnormal stages/patterns of a seizure, such as pre-ictal (EEG changes preceding a seizure) and ictal (EEG changes during a seizure).
\end{abstract}

Keywords: Electroencephalographic time series $\cdot$ Epilepsy $\cdot$ Complex networks · Quantile graphs

\section{Introduction}

Epilepsy is a neurological disorder characterized by the presence of recurring seizures that affects nearly $1 \%$ of the general population [1]. Sudden and abrupt seizures that cause momentarily lapses of consciousness can have significant impact on the daily life of sufferers. Thus, epileptic seizure detection would help these people to have a normal life.

Like many other neurological disorders, epilepsy can be assessed by the electroencephalogram technic. Visual inspection of the EEG data has not yet led to the detection of all characteristic changes preceding seizure onsets. Moreover, detection of seizures by visual inspection of the EEG even by a trained neurologist is challenging for a variety of reasons such as the excessive presence 
of myogenic artifacts [3]. Hence, several studies have focused on the detection of epilepsy from EEG signals using automatic analysis like methods that detect and quantify non-linear mechanisms and thereby better reflect the characteristics of the EEG signals [3,5]. Therefore, time-domain methods $[4,5,16]$, frequencydomain methods $[13,15]$ and Wavelet-domain methods $[12,14]$ have been recently proposed. However, there is still considerable research toward developing novel methods to quantify or capture additional information in EEG time series in new ways.

In the last two decades, research on complex networks became the focus of widespread attention, with developments and applications spanning different scientific areas, from sociology and biology to physics [2]. One of the reasons behind the growing popularity of complex networks is that almost any discrete structure can be suitably represented as a graph, whose features may be then characterized, analyzed and, eventually, related to its respective dynamics [9]. Recently an approach has been proposed for mapping a time series into a complex network representation, based on the concept of transition probabilities, resulting in a so-called "quantile graph" (QG) $[7,8]$. This study has shown that distinct features of a time series can be mapped into networks with distinct topological properties, opening the door to the analysis of discrete, time-ordered data sets with mathematical tools usually used in the study of geometric shapes and topological spaces. Here we show that the complex network theory can be effectively used to the problem of detecting differences in EEG signals of patients with and without epilepsy symptoms. It is important to mention that our method does not require assumptions about stationarity, length of the signal, and noise level. The rest of this paper is organized as follows. After this Introduction, in Sect. 2 it is described the QG method for mapping a time series into a network. In Sect. 3 it is described the data set used in this study. Results are presented and discussed in Sect. 4 while an overall conclusion is given in Sect. 5 .

\section{Methods}

Let $\mathcal{M}$ be a map from a time series $X \in \mathcal{T}$ to a network $g \in \mathcal{G}$, with $X=$ $\{x(t) \mid t \in \mathbb{N}, x(t) \in \mathbb{R}\}$ and $g=\{\mathcal{N}, \mathcal{A}\}$ being a set of nodes $\mathcal{N}$ and $\operatorname{arcs} \mathcal{A}$. $\mathcal{M}$ assumes a simple discretization of $X$ that is not sensitive to the distribution of its values. Specifically, once the $Q$ quantiles have been identified, $\mathcal{M}$ assigns each quantile $q_{i}$ to a node $n_{i} \in \mathcal{N}$ in the corresponding network. Two nodes $n_{i}$ and $n_{j}$ are connected with a weighted $\operatorname{arc} n_{i}, n_{j}, w_{i j}^{k} \in \mathcal{A}$ whenever two values $x(t)$ and $x(t+k)$ belong respectively to quantiles $q_{i}$ and $q_{j}$, with $t=1,2, \ldots, T$ and the time differences $k=1, \ldots, k_{\max }<T$. For an illustration of the QG method for $k=1$, see Fig. 1 .

Weights $w_{i j}^{k}$ are simply given by the number of times a value in quantile $q_{i}$ at time $t$ is followed by a point in quantile $q_{j}$ at time $t+k$, normalized by the total number of transitions. Repeated transitions through the same arc increase the value of the corresponding weight. With proper normalization, the weighted adjacency matrix becomes a Markov transition matrix $\mathbf{W}_{k}$, with $\sum_{j}^{Q} w_{i j}^{k}=1$. 
Time series, $\mathrm{X}$

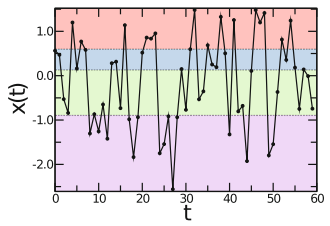

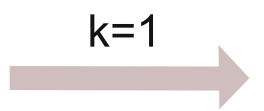

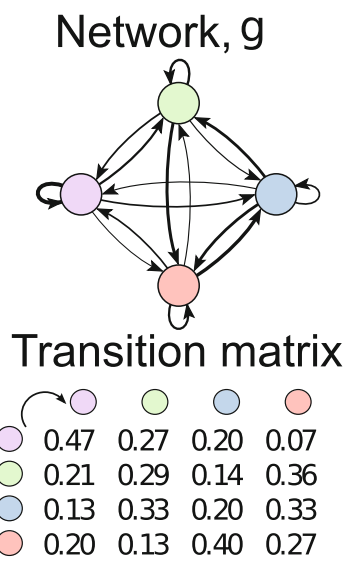

Fig. 1. Illustration of the QG method for $k=1$. A white Gaussian noise $X$ with $T=60$ time points is split into $Q=4$ quantiles (colored shading) and each quantile $q_{i}$ is assigned to a node $n_{i} \in \mathcal{N}$ in the corresponding network $g$. Two nodes $n_{i}$ and $n_{j}$ are then connected in the network with a weighted $\operatorname{arc}\left(n_{i}, n_{j}, w_{i j}^{k}\right) \in \mathcal{A}$ where the weight $w_{i j}$ of the arc is given by the probability that a point in quantile $q_{i}$ is followed by a point in quantile $q_{j}$. Repeated transitions between quantiles results in arcs in the network with larger weights (represented by thicker lines) and therefore higher values in the corresponding transition matrix. (Color figure online)

Since $\mathcal{M}$ is insensitive to the distribution of values of $X$, the map only requires the specification of the parameter $Q$, the number of quantiles/nodes, with $Q$ being typically much smaller than $T$ [8].

We previously showed [7] that with $\mathbf{W}_{k}$ in hand, it is possible to perform a random walk on the quantile graph (QG) $g$. After $l=L$ jumps of length $\delta_{l, k}(i, j)=|i-j|$, with $i, j=1, \ldots, Q$ being the node indices as defined by $\mathbf{W}_{k}$, the mean jump length $\Delta(k)$ can be computed as follows:

$$
\Delta(k)=\frac{1}{L} \sum_{l=1}^{L} \delta_{l, k}(i, j) .
$$

The mean jump length $\Delta(k)$ can be computed directly from the transition matrix $\mathbf{W}_{k}$ [10], an approach less time-consuming, especially for networks with many nodes. In this case, $\Delta(k)$ given by:

$$
\Delta(k)=\frac{1}{Q} \operatorname{tr}\left(\mathbf{P} \mathbf{W}_{k}^{T}\right) .
$$

where $\mathbf{W}_{k}^{T}$ is the transpose of $\mathbf{W}_{k}, \mathbf{P}$ is a $Q \times Q$ matrix with elements $p_{i, j}=|i-j|$, and $t r$ is the trace operation.

Previous works have shown that time series with different properties are mapped into complex networks with different topologies. For example, we have found an association between periodic time series and regular networks, random 
time series and random networks, pseudo-periodic time series and small-world networks [8]. Moreover, we have shown that the bifurcation cascades of two well known unimodal maps - the Logistic and Quadratic Maps - are mapped through the QG method into networks whose topological characteristics mimic the main features of their period-doubling route to chaos as a forcing parameter varies continuously [6]. Finally, we recently showed that the QG method permits to quantify features such as long-range correlations or anti-correlations and it can be used to estimate the Hurst exponent of fractional motions and noises [7].

\section{Data}

In this study, we use an artifact free EEG database, provided by the University of Bonn and freely available online [5]. This database has been widely used for EEG feature extraction and classification in the literature [3]. Our data consists of five sets (denoted A, B, C, D and E), each containing 100 single-channel EEG segments, with a duration of $23.6 \mathrm{~s}$, and a sampling rate of $173.61 \mathrm{~Hz}$. Sets A and B consist of surface EEG recordings from healthy, awake volunteers with eyes open (A) and closed (B), respectively. Sets C and D correspond to intracranial

(A)

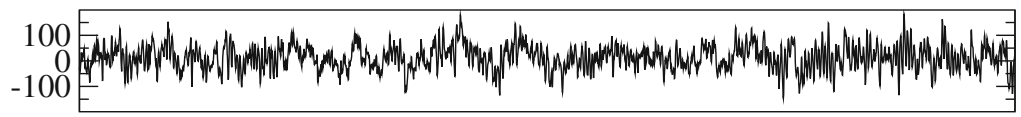

(B)

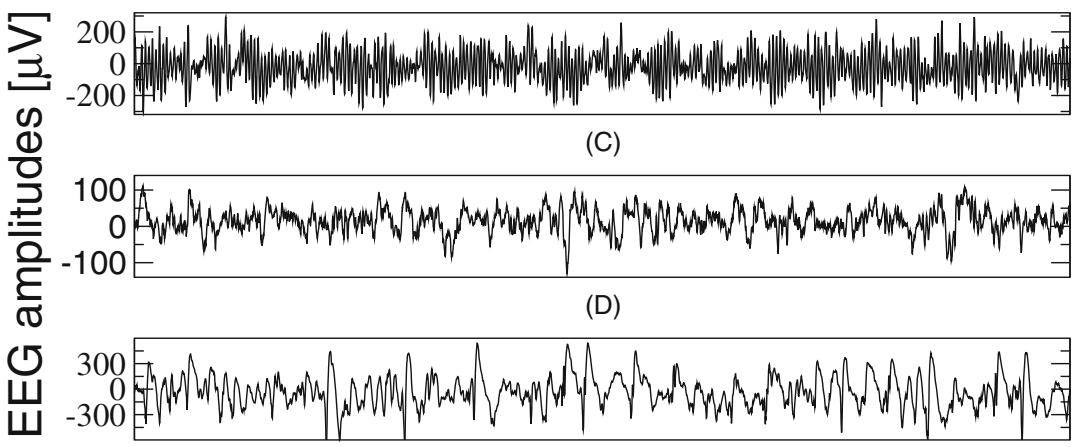

(E)

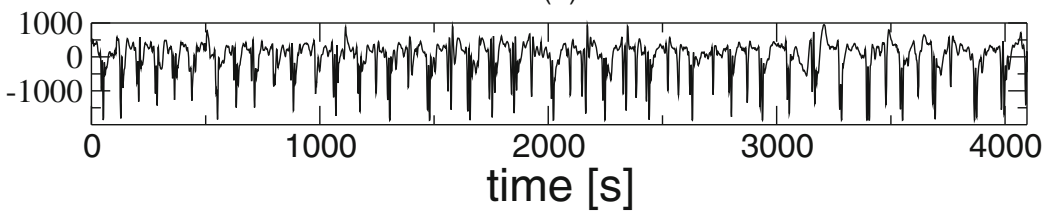

Fig. 2. Typical EEG signals from each of the five sets; from top to bottom: A (healthy, eyes open), B (healthy, eyes closed), C (epileptic, opposite zone), D (epileptic, epileptogenic zone) and E (seizure). 
EEG signals from epileptic patients recorded during seizure-free intervals at the epileptogenic zone (D) and at the hippocampal formation in the opposite hemisphere of the brain (C). Finally, intracranial EEG signals in set E contain seizure activity, measured at sites exhibiting ictal activity.

Figure 2 shows samples from sets A, B, C, D and E. EEG time series recorded extracranially during the relaxed state of healthy subjects with eyes closed (Fig. 2(B)) show a predominant physiological rhythm, the so-called "alpha rhythm" in a frequency range of $8-13 \mathrm{~Hz}$, an activity which is most pronounced at the back of the head. In contrast, broader frequency characteristics are obtained for open eyes (Fig. 2(A)). During a seizure free interval, the EEG recorded from within the epileptogenic zone (Fig. 2(D)) is often characterized by intermittent occurrences of interictal epileptiform activities. Investigation of these steep, sometimes rhythmic high amplitude patterns in EEG recordings contributes to the localization of the epileptogenic zone. Fewer and less pronounced interictal epileptiform activities can be found at recording sites distant from the epileptogenic zone (Fig. 2(C)). As for the EEG recorded during epileptic seizures (Fig. 2(E)), termed ictal activity, it is almost periodic and of high amplitude, resulting from the hypersynchronous activity of large assemblies of neurons [11].

\section{Results}

We apply the QG algorithm to the problem of differentiating (a) epileptic from normal data, (b) EEG changes preceding a seizure and (c) EEG changes during a seizure. Unless when indicated otherwise, the values $Q=30, T=4096$ and $k=1,2, \ldots, 100$ have been used in the computations. Therefore, we mapped 500 signals into 50,000 quantile graphs and we obtained 50,000 QG transition matrices. After that, for each set and for a given $k$ we took the median over all matrices and we obtained a QG transition matrix of medians. We finally computed $\Delta(k)$ versus $k$ using Eq. (2) for all sets (Fig. 3). Observe that the curves for healthy (A and B) and epileptic (C and D) patients form two distinct clusters with maximum separation at approximately $k=4$. For $k>30$, correlations between QG nodes disappear, and all curves almost merge into one.

Figure 4 displays boxplots of $\Delta(k)$, computed over 100 segments, for sets $\mathrm{A}, \mathrm{B}, \mathrm{C}, \mathrm{D}$ and $\mathrm{E}$, and $k=4$. Note how the QG method permits a robust discrimination between healthy and epileptic patients, even more when the EEGs are taken from healthy subjects with the eyes closed. Comparing sets B (healthy, eyes closed) and $\mathrm{C}$ (epileptic, opposite zone), there is a statistically significant difference between the two sample means of $\mu_{B}-\mu_{C}=4.5389$, with a $95 \%$ confidence interval of $[4.2989,4.7790], t(d f=195.3079)=37.2897$ and $p \leq 0.05$. For sets A (healthy, eyes open) and D (epileptic, epileptogenic zone), the less favorable comparison, we still have a statistically significant difference between the two sample means, with $\mu_{A}-\mu_{D}=2.9658$, with a $95 \%$ confidence interval of $[2.6689,3.2625], t(d f=191.8404)=19.7085$ and $p \leq 0.05$.

Figure 5 displays QG transition matrices of medians for $k=4$. Observe how well the QG topology mimics the properties of the original time series. For 


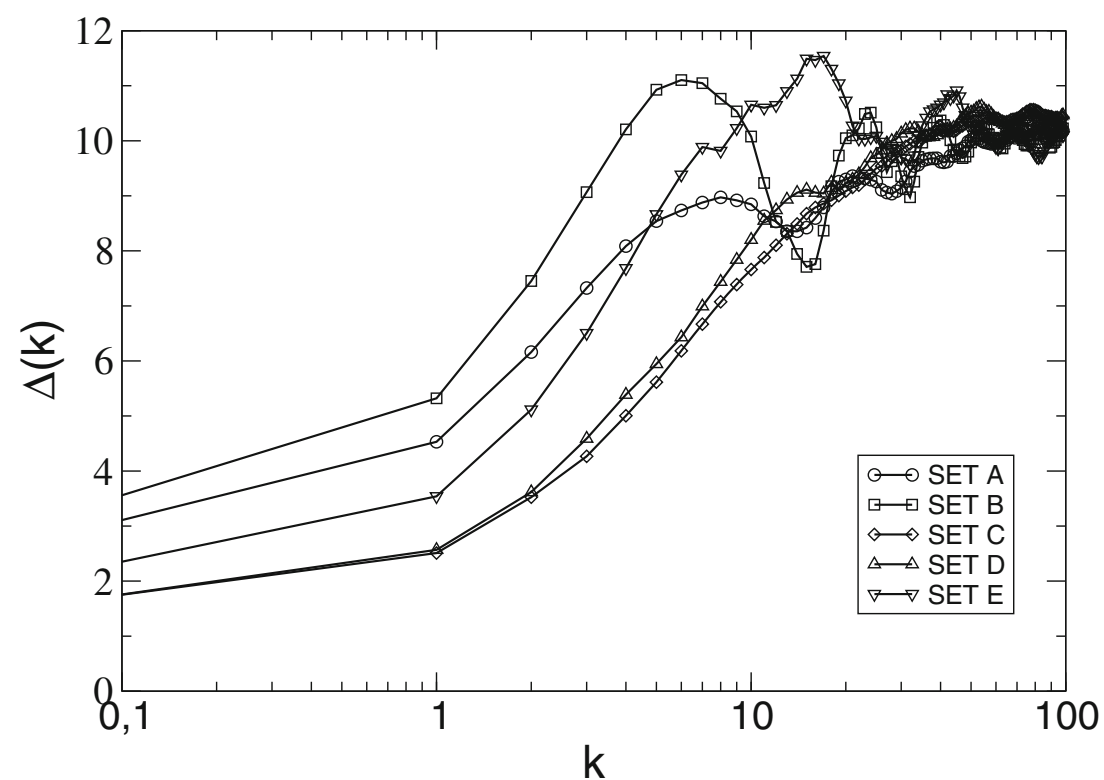

Fig. 3. $\Delta(k)$ versus $k$ for sets A (healthy, eyes open), B (healthy, eyes closed), C (epileptic, opposite zone), D (epileptic, epilectogenic zone) and E (seizure).

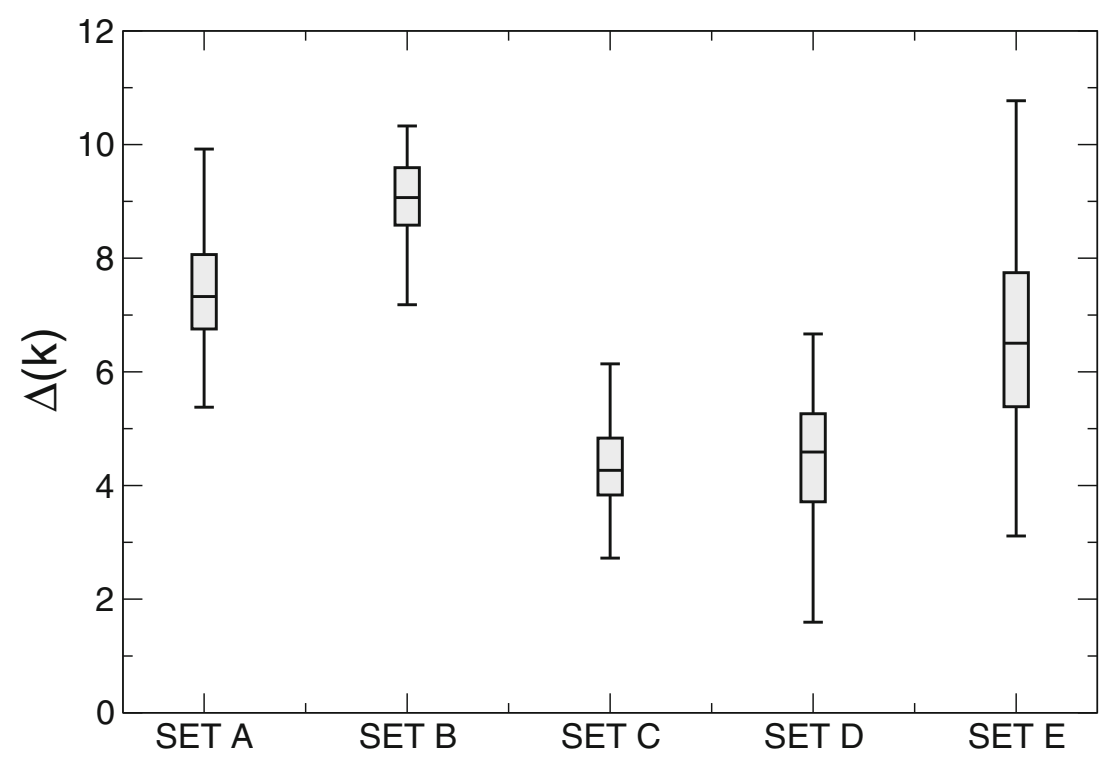

Fig. 4. Boxplots of $\Delta(k)$, computed over 100 segments each, for sets A (healthy, eyes open), B (healthy, eyes closed), C (epileptic, opposite zone), D (epileptic, epilectogenic zone) and $\mathrm{E}$ (seizure), and $k=4$. 

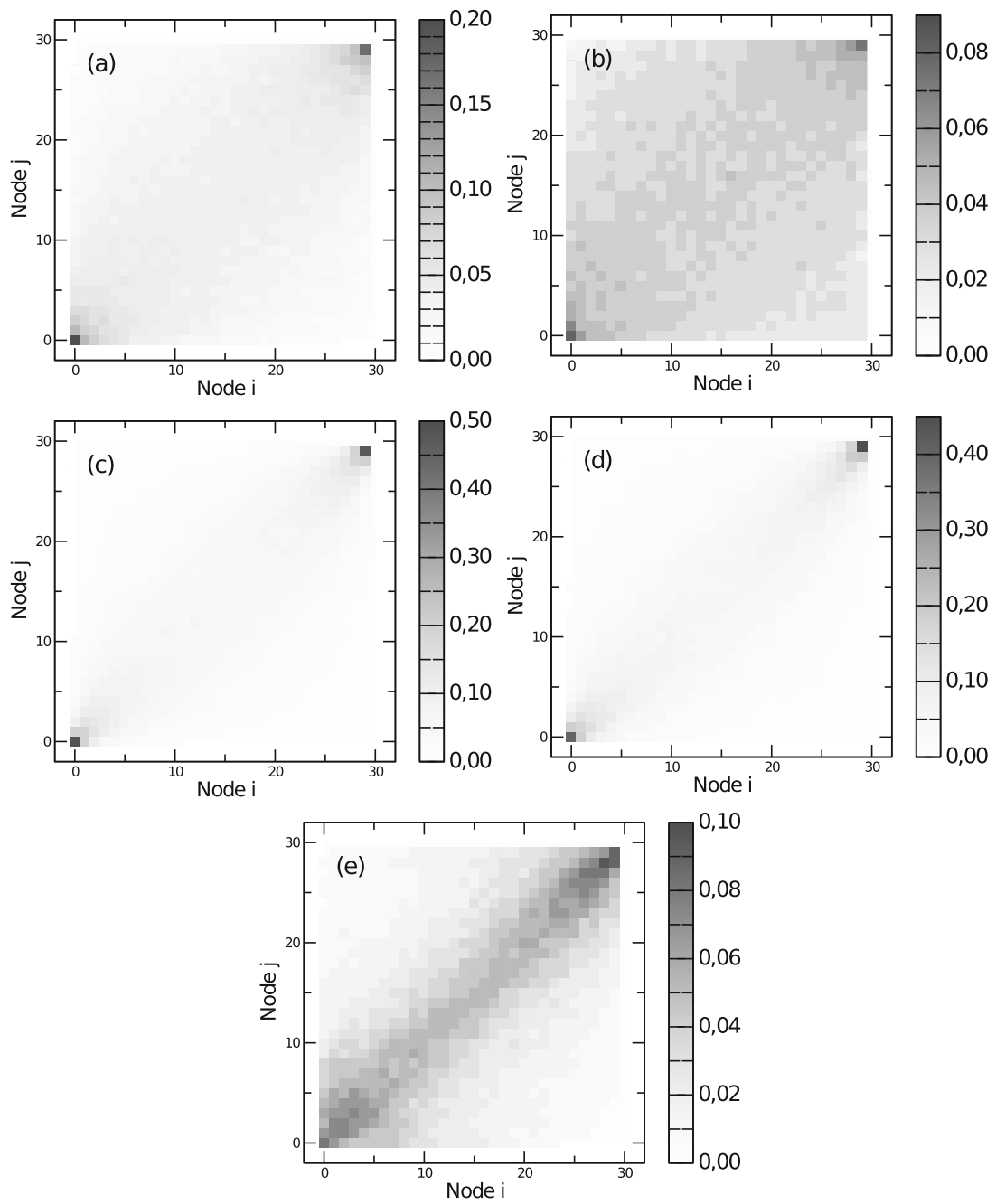

Fig. 5. QG transition matrices for sets A (healthy, eyes open), B (healthy, eyes closed), C (epileptic, opposite zone), D (epileptic, epileptogenic zone) and E (seizure), and $k=4$.

healthy subjects from set B (Fig. 5(b)), the weights in the corresponding $\mathbf{W}_{4}$ are more uniformly distributed along its columns and rows when compared with $\mathbf{W}_{4}$ for healthy subjects from set A (Fig. 5(a)). For unhealthy subjects from set D (Fig. 5(d)), the higher weights in the corresponding $\mathbf{W}_{4}$ are concentrated in its peripherical quantiles due to the rhythmic high amplitude patterns found in the corresponding time series. Although Fig. 5(c) and (d) are very alike, the fewer and less pronounced interictal epileptiform activities found in set $\mathrm{C}$ (when compared to set D) produce a QG transition matrix with heavier weights. Finally, 
the high amplitude and quasiperiodic patterns found in set E are mapped in a QG transition matrix with its weights mainly distributed over the secondary diagonal (Fig. 5(e)).

\section{Conclusions}

A novel method for classifying EEG data by using the concept of quantile graphs was presented. We have shown that it can not only differentiate epileptic from normal data, but also distinguish the different abnormal stages/patterns of a seizure, such as pre-ictal (EEG changes preceding a seizure) and ictal (EEG changes during a seizure). These results attest that the QG method is a useful tool for the analysis of nonlinear dynamics and able to detect differences in the data structures of physiological signals of healthy and unhealthy subjects. The QG method is numerically simple and has only one free parameter, $Q$, the number of quantiles/nodes. The number of quantiles $Q$ defines the partitioning level of the amplitude range of the original time series and its selection involves a trade-off between information loss and computational burden.

Acknowledgments. A.S.L.O. Campanharo acknowledges the support of FAPESP: 2013/19905-3. The support of Conselho Nacional de Desenvolvimento Científico e Tecnológico (CNPq) (Brazil) is acknowledged by F.M. R. All figures were generated with PyGrace (http://pygrace.github.io/) with color schemes from Colorbrewer (http:// colorbrewer.org).

\section{References}

1. Seizures and epilepsy: hope through research (2004). http://www.ninds.nih.gov/ disorders/epilepsy/detail_epilepsy.htm

2. Albert, R., Barabási, A.L.: Statistical mechanics of complex networks. Rev. Modern Phys. 74, 47 (2002)

3. Alotaiby, T.N., Alshebeili, S.A., Alshawi, T., Ahmad, I., El-samie, F.E.A.: EEG seizure detection and prediction algorithms: a survey. EURASIP J. Adv. Sig. Process. 183, 1-21 (2014)

4. Andrzejak, R.G., Lehnertz, K., Mormann, F., Rieke, C., David, P., Elger, C.E.: Indications of nonlinear dynamics and finite-dimensional structures in time series of brain electrical activity: dependence on recording region and brain state. Phys. Rev. E 64, 061907 (2001)

5. Andrzejak, R.G., Schindler, K., Rummel, C.: Nonrandomness, nonlinear dependence, and nonstationarity of electroencephalographic recordings from epilepsy patients. Phys. Rev. E 86, 046206 (2012)

6. Campanharo, A., Ramos, F.M.: Quantile graphs for the characterization of chaotic dynamics in time series. In: WCCS 2015 - IEEE Third World Conference on Complex Systems. IEEE (2016)

7. Campanharo, A., Ramos, F.M.: Hurst exponent estimation of self-affine time series using quantile graphs. Physica A 444, 43-48 (2016)

8. Campanharo, A., Sirer, M.I., Malmgren, R.D., Ramos, F.M., Amaral, L.A.N.: Duality between time series and networks. PLoS ONE 6, e23378 (2011) 
9. Costa, L.F., Rodrigues, F.A., Travieso, G., Villas, P.R.: Characterization of complex networks. Adv. Phys. 56, 167-242 (2007)

10. Doescher, E., Campanharo, A.S.L.O., Ramos, F.M.: Quantile graphs: exact results and applications (2017, in preparation)

11. Güler, I., Übeyli, E.D.: Expert systems for time-varying biomedical signals using eigenvector methods. Expert Syst. Appl. 32, 1045-1058 (2007)

12. Guo, L., Rivero, D., Pazos, A.: Epileptic seizure detection using multiwavelet transform based approximate entropy and artificial neural networks. J. Neurosci. Meth. 193, 156-163 (2010)

13. Khamis, H., Mohamed, A., Simpson, S.: Frequency-moment signatures: a method for automated seizure detection from scalp EEG. Clin. Neurophysiol. 124, 2317$2327(2013)$

14. Liu, Y., Zhou, W., Yuan, Q., Chen, S.: Automatic seizure detection using wavelet transform and svm in long-term intracranial EEG. EEE Trans. Neural Syst. Rehabil. Eng. 20, 749-755 (2012)

15. Rana, P., Lipor, J., Lee, H., Van Drongelen, W., Kohrman, M.H., Van Veen, B.: Seizure detection using the phase-slope index and multichannel ECoG. IEEE Trans. Biomed. Eng. 59, 1125-1134 (2012)

16. Vlachos, I., Kugiumtzis, D.: Nonuniform state-space reconstruction and coupling detection. Phys. Rev. E 82, 016207 (2010) 